Transportation Research Forum

Comparison of TRANSIMS' Light Duty Vehicle Emissions with On-Road Emission Measurements Author(s): Mansoureh Jeihani, Kyoungho Ahn, Antoine G. Hobeika, Hanif D. Sherali, and Hesham A.

Rakha

Source: Journal of the Transportation Research Forum, Vol. 45, No. 1 (Spring 2006), pp. 87-99

Published by: Transportation Research Forum

Stable URL: http://www.trforum.org/journal

The Transportation Research Forum, founded in 1958, is an independent, nonprofit organization of transportation professionals who conduct, use, and benefit from research. Its purpose is to provide an impartial meeting ground for carriers, shippers, government officials, consultants, university researchers, suppliers, and others seeking exchange of information and ideas related to both passenger and freight transportation. More information on the Transportation Research Forum can be found on the Web at www.trforum.org. 


\section{Comparison of TRANSIMS' Light Duty Vehicle Emissions with On-Road Emission Measurements}

The Transportation Analysis and Simulation System, TRANSIMS, contains a vehicle emissions module that estimates tailpipe emissions for light and heavy-duty vehicles and evaporative emissions for light-duty vehicles. This paper describes and validates the TRANSIMS emission module and compares its emission estimates to on-road emission-measurements and other state-of-the-art emission models. The trend of the emissions estimated in thirteen different runs in each model are compared. The results indicate that the TRANSIMS model provides consistent trends of estimated carbon monoxide (CO) and hydrocarbons (HC) with field data trends and inconsistent trends of estimated nitrogen lxides (NOx). However, the magnitude of the emission estimated in TRANSIMS is closer to the field data than for other models.

\section{by Mansoureh Jeihani, Kyoungho Ahn, Antoine G. Hobeika, Hanif D. Sherali, and Hesham A. Rakha}

\section{INTRODUCTION}

Emissions from vehicle sources have a major effect on urban air quality. According to the Clean Air Act Amendments of 1990 (CAAA), non-attainment areas are required to submit emission estimates for all proposed trafficimprovement projects. Therefore, a reliable and accurate emission estimation model is needed. Traditional emission estimation modeling techniques are not appropriate for small-scale traffic improvement projects. These models use an aggregate representation of traveler behavior and estimate emissions based on typical driving cycles using vehicle miles traveled and average speeds, supplemented by corrections for cold-start, evaporation, and high emitting vehicles. However, vehicle emissions are usually produced from off-cycle driving, vehicles malfunctioning, and climbing steep grades, which are not considered in these models. Hence, these techniques underestimate emissions because they cannot accurately estimate impacts. In addition, existing models do not estimate the effects of green wave signalization that allows vehicles to be in phase with the green lights by traveling at or near the speed limit. To our knowledge, the Transportation Analysis and Simulation System (TRANSIMS) is the only model that has the green wave effect estimation function.
To improve emissions estimation procedures, microscopic emissions modeling has been introduced in the literature. However, in general the transportation modeling community and the air quality modeling community have worked separately on this issue. Two models that appear to have addressed this issue are the INTEGRATION software (Rakha and Ahn 2004) and the Transportation Analysis and Simulation System (TRANSIMS). TRANSIMS combines a cellular automata vehicle movement model (micro-simulator) with a microscopic second-by-second tailpipe emissions model based on the Comprehensive Modal Emissions Model (CMEM) model.

The objective of this paper is to describe and validate the TRANSIMS Emission module using the On-Road Emission Measurement (OEM). OEM is a new technology that collects vehicle emissions during real driving conditions using a portable instrument, and is a viable alternative for collecting emission data in the field. The emissions estimated by TRANSIMS are compared to those estimated by other emissions estimation models, MOBILE6, CMEM, and VT-Micro model.

Roden (2005) reported that the emissions estimated by MOBILE6 using simulation output generated by TRANSIMS were significantly different from those produced using traditional modeling techniques. This paper extends Roden's 
study by validating the TRANSIMS emission estimates against field data and other state-ofthe-art emission models.

The remainder of the paper is organized as follows. It first briefly explains the emissions estimation model within MOBILE6, CMEM, VT-Micro, and TRANSIMS. It also describes the algorithmic procedure of the TRANSIMS Emission Estimator module, applies the models to a real-world network, Blacksburg, Virginia, and compares the results with OEM data. The trend obtained from thirteen runs using MOBILE6, CMEM, VT-Micro, and TRANSIMS are compared with the trend in field data. Finally, a summary of the paper and the conclusions are provided.

\section{EMISSIONS ESTIMATION MODEL DESCRIPTION}

This section briefly describes the emissions models compared in this study (e.g., MOBILE6, CMEM, and VT-Micro models). Since the focus of the paper is on the TRANSIMS Emissions Estimator, it is explained in greater detail.

CMEM, VT-Micro, and TRANSIMS are microscopic emissions models, while MOBILE6 utilizes a macroscopic approach to estimate mobile source emissions. However, this research includes the result of MOBILE6, as it is currently the only emission model utilized by U.S. government and local agencies to estimate mobile source emissions. Thus, the comparison with MOBILE6 can provide overall evaluation of absolute vehicle emission value that is estimated from TRANSIMS.

\section{MOBILE Model}

The MOBILE model was developed by the Environmental Protection Agency (EPA) to predict vehicle emissions based on average speeds of trips, specifically emissions inventories for large regional areas. It was built using regression coefficients based on federal test procedure (FTP) bag emission measurements, to predict emissions inventories for large regional areas.

In an effort to address some problems in the Mobile model, MOBILE6 was introduced.
This model is based on recent vehicle emission testing data collected by the EPA, California Air Resource Board (CARB), automobile manufacturers, and Inspection and Maintenance (I/M) tests from several states. It also models the impact of different petroleum refiners on vehicle emissions.

A major characteristic of MOBILE6 is the addition of "off-cycle emissions," which involves aggressive driving with the air conditioning operating. This aggressive driving behavior is not included in the FTP drive cycle, but it is included in the Supplemental FTP cycle (which applies to model year 2000 and newer vehicles). As drive cycles used in MOBILE6 include operations at high speeds and high accelerations, the model produces significantly higher pollutants in comparison to MOBILE5. MOBILE6 estimates emission factors based on different roadway types (e.g., highways, arterials, and locals). These emission factors can be adjusted based on vehicle testing over a series of facility cycles for different facility types and different average speeds. MOBILE6 estimates emission factors from the starting and running portions of the trip separately. Coldstart emissions are calculated using FTP bag1 (e.g., cold-start emissions) and FTP bag3 (e.g., hot-start emissions).

Other significant enhancements to MOBILE6 include (i) dramatic reductions in vehicle emissions as vehicles age and accumulate mileage, (ii) control of off-cycle emissions with the Supplemental FTP (SFTP) drive cycle, (iii) the inclusion of evaporative diurnal emission factors estimated from real-time diurnal test data previously unavailable, (iv) the revision of oxygenated fuel effects, (v) the revision of I/M program effects on vehicle emissions, (vi) the addition of off-cycle $\mathrm{NO}_{\mathrm{x}}$ emissions for heavyduty diesel vehicles, (vii) the effects of in-use fuel sulfur content on all emissions, and (viii) the effects of national low-emission vehicles (NLEV) and Tier 3 standards (EPA 2001 and NRC 2000). It should be noted, however, that in spite of these model improvements, the MOBILE6 model generates identical emission estimates for trips with identical average speeds if the roadway facility is the same. 
CMEM Model

The Comprehensive Modal Emissions Model (CMEM), which is one of the newest power demand-based emission models, was developed by Barth et al (1997) under contract with the National Cooperative Highway Research Program (NCHRP). CMEM is an improved modal emission model for light-duty vehicles (LDV). It calculates tractive power by taking into account engine-friction losses, rolling resistance, wind resistance, changes in kinetic energy, changes in potential energy, and the power necessary to drive accessories such as air conditioning. With engine power known, CMEM calculates the rate of fuel consumption and tailpipe emissions and considers enrichment, enleanment, and stoichiometric operations, as well as cold-start operations.

CMEM estimates light-duty vehicles (LDV) and light-duty trucks (LDT) emissions as a function of a vehicle's operating mode. The term "comprehensive" is utilized to reflect the ability of the model to predict emissions for a wide variety of LDVs and LDTs in various operating states (e.g., properly functioning, deteriorated, malfunctioning). For the test data, both engine-out and tailpipe emissions of over 300 vehicles, including more than 30 high emitters, were measured second-bysecond over three driving cycles, including the Federal Test Procedure (FTP), US06, and the Modal Emission Cycle (MEC). CMEM predicts second-by-second tailpipe emissions and fuel-consumption rates for a wide range of vehicle/technology categories. The model is based on a parameterized, physical approach that decomposes the entire emission process into components corresponding to the physical phenomena associated with vehicle operation and emission production. Vehicle and operation variables (e.g., speed, acceleration, and road grade) and model-calibrated parameters (e.g., cold-start coefficients and engine-friction factor) are utilized as input data (Barth et al. 2000).

To compare the results of the CMEM model with measured emissions and other models, CMEM vehicle category 11 was utilized since the test vehicle, a 1999 Crown Victoria, falls within this category. The speed profiles of the test runs were utilized as input data for the purposes of comparison.

\section{VT-Micro Model}

The VT-Micro emission model is a nonlinear regression model that utilizes a multidimensional polynomial model structure that is described in great detail in the literature (Rakha et al. 2004a; Rakha et al. 2004b; Rakha and Ahn 2004; Rakha et al. 2002). This multiple regression model relates the dependent variables (instantaneous emission estimates) to a set of quantitative independent variables, namely, instantaneous speed and acceleration levels. The regression model includes a combination of linear, quadratic, and cubic speed and acceleration terms. While a more detailed description of the derivation of the model is provided in the literature (Ahn et al. 2002), it is sufficient to note that the structure of the model involves a logarithmic transformation of a dualregime third-order polynomial. The logarithmic transformation of the emission measurements ensures non-negative model predictions and accurate model predictions in the low-speed and low-acceleration range, with their associated low-emission rates. Furthermore, the use of a dual-regime model ensures a better fit to the data over the full range of the vehicle-operation envelope. Specifically, the use of a separate regime for the deceleration mode of operation allows the model to capture the higher vehicleemission rates at higher vehicle speeds during a vehicle-deceleration maneuver.

A common problem with multi-regime models is the function's discontinuity at the regime boundaries. To overcome this potential problem, the emission data for the deceleration regime was shifted by the value of the $y$ intercept for the acceleration regime to ensure that both intercepts were equal. It should be noted that the models were confined to speed and acceleration levels within the envelope of the OEM data. This constraint resulted from the inherent limitation of any model to extrapolate response values beyond the boundaries used in developing the model. While vehicles may travel faster than the maximum speed of the model, which is $129 \mathrm{~km} / \mathrm{h}(80 \mathrm{mi} / \mathrm{h})$, it is highly unlikely to observe speeds outside this range. 
Consequently, the envelope of data coverage appears to cover the full range of typical vehicle operation. However, in cases in which the speed/acceleration data fall outside the model envelope, the model developers recommend using boundary speed and acceleration levels to ensure realistic vehicle measure of effectiveness (MOE) estimates.

This study utilized the VT-OEM and VT LDV5 models. The VT-OEM model was developed using the test vehicle emission data that were gathered by a Portable On-Road Emission Measurement System. The VT LDV5 model represents the emissions of an average vehicle based on in-laboratory data from the U.S. Environmental Agency. This study uses VT LDV5, since the test vehicle falls within the desired category.

The VT-Micro model has been incorporated within the INTEGRATION microscopic traffic assignment and simulation software (Rakha and Ahn, 2004). This software combines a vehicle dynamics model, a steady-state car-following logic, and collision-avoidance constraints together with the VT-Micro model to estimate vehicle emissions.

\section{TRANSIMS Emissions Estimator}

The Transportation Analysis and Simulation System (TRANSIMS) is part of the Travel Model Improvement Program sponsored by the U.S. Department of Transportation, the Environmental Protection Agency, and the Department of Energy. It is a set of integrated models designed to forecast travel demands at the individual-user level rather than at the zonal aggregate level. It simulates second-bysecond movements of travelers on the network. Then, it estimates emissions considering coldstarts, enrichment cycles, grades, and vehicle malfunction.

TRANSIMS consists of six modules as follows. The Population Synthesizer estimates the number of synthetic households, their characteristics, and their locations on the network. The Activity Generator determines an activity list, activity times, and activity locations for each synthetic traveler. The Route Planner computes combined route and mode trip plans to accomplish the desired activities of each individual. The Microsimulator performs a regional microsimulation of vehicle interactions and computes at every second the operating status, including locations and speeds, of all vehicles throughout the simulation period. The Feedback Controller manages the feedback of information among the Activity Generator, the Route Planner, and the Traffic Microsimulator modules of TRANSIMS. The Emissions Estimator forecasts the nature, amount, and location of motor vehicle emissions using the vehicle information generated in the Microsimulation module.

The Emissions Estimator in TRANSIMS produces estimates for tailpipe emissions from light-duty vehicles (LDVs), tailpipe emissions from heavy-duty vehicles (HDVs), and evaporative emissions as a function of vehicle fleet composition, fleet status, and fleet dynamics. This paper focuses only on tailpipe emissions from LDVs. The Emissions Estimator module requires information regarding the fleet composition developed from the Population Synthesizer, inspection and maintenance test results obtained from local and national databases, and traffic patterns produced by the Traffic Microsimulator module. The emission inventory obtained from TRANSIMS form the basis for the computation of pollutant concentrations in a metropolitan area based on atmospheric conditions, local transport and dispersion, and chemical reactions.

The Microsimulator adopts a cellularautomata principle that gives the vehicle position in units of cells, velocity expressed in cells per second, and acceleration in units of cells per second per second. Since the cell size is $7.5 \mathrm{~m}$ the resulting movement in 16-mph increments is too coarse to estimate emissions. Therefore, smooth vehicle trajectories are generated and used in the emissions estimation. The output of the Microsimulator is aggregated into 30-meter segments, $7.5 \mathrm{~m} / \mathrm{s}$ speed bins, and over an hour. This converted output from the Microsimulator is used as the major input to the Emissions Estimator. The following algorithm is used in the Emissions Estimator module.

In TRANSIMS, empirical information on power demands is used to estimate the emissions due to the lack of information describing the range of driving behavior under 
various circumstances. Three sets of empirical data are used as follows. The first is the CMEM emissions array. There are two CMEM arrays; one reflects emissions at constant power and another reflects differences in emissions associated with changes in power from one second to the next. These arrays are calculated from CMEM 1.2 by performing extensive tests on over 300 vehicles chosen to represent the major types of emitters in the existing LDV fleets.

The EPA three-cities data is the second empirical data used in TRANSIMS. It is used to estimate the distribution of high-power and hard-braking events. The three-cities study collects a survey of driving behavior in three different cities for a total of more than 200 different vehicles. It gives the cumulative distribution of acceleration for hard acceleration (VA $>50$ mile per hour squared per second, mph2ps), hard braking (VA $<-50 \mathrm{mph} 2 \mathrm{ps}$ ), and insignificant acceleration $(-50<\mathrm{VA}<50)$, where VA is the velocity-acceleration product and is used to represent power.

The third empirical data used in TRANSIMS is the California Air Resource Board (CARB) data. The CARB data is a collection of vehicle trajectories on freeways and arterials having different levels of congestion. It is also used for deriving calibration constants. CARB sponsored a chase-car study in Los Angeles to collect the data. The resulting data gives the distribution of velocities and accelerations on ramps.

Using the three sets of empirical data and the converted Microsimulator output, emissions are estimated in TRANSIMS as follows. The converted Microsimulator output is used to calculate the number of vehicles for each 4-mph speed bin and 10-mph2ps power bin. This is done to obtain an array having the same structure as that of the CMEM arrays. The choice of emission arrays to have 4-mph speed bins and 10-mph2ps power bins is driven by the sensitivity of emissions to power and speed. The range of power is considered from -150 to $180-\mathrm{mph} 2 \mathrm{ps}$ in $10-\mathrm{mph} 2 \mathrm{ps}$ increments — for a total of 34 power bins. The speeds are considered to range from $2 \mathrm{mph}$ to $78 \mathrm{mph}$ in 4 mph increments - for a total of 20 speed bins. A flow chart of the TRANSIMS overall emission estimator module is shown in Figure 1.

The first step estimates the population of vehicles in each 4-mph speed cell from the population in the $7.5 \mathrm{~m} / \mathrm{s}$ speed bins for each link segment. This is carried out by constructing a continuous distribution of the number of vehicles by speed group by assuming that the continuous speed distribution within each speed bin can be approximated by a linear function of speed. The result is also used to calculate the standard deviation of speed, the average square of the speed, and the average cube of the speed in each segment.

There is a nonlinear relationship between power and emissions. The average power in each segment is estimated using the average cube of the speed. The probability of hard acceleration, hard braking, and insignificant acceleration is influenced by the average power and also by the standard deviation of speed. A simple linear relationship is found between the gradient of the average cube of the speed or the standard deviation of the speed and the probability of hard acceleration. Therefore, the probability of hard acceleration or hard braking is calculated using the average cube of the speed and the standard deviation of speed. Also, the total flux (the first moment of speed) is broken into thirds and the probability of hard acceleration (braking) is calibrated for each third separately. These are distributed over the power bins using the cumulative VA distributions from the EPA's three-cities data. An adjustment is made to represent the emissions associated with the step change in power.

Fleet composition is developed from vehicle registration data, inspection, and maintenance testing, or from data developed from the EPA's Mobile5 model runs. The registration data is used to produce vehicle populations in each of the $23 \mathrm{LDV}$ vehicle categories. These categories include factors such as low or high engine-to-weight ratio, car or truck mileage above or below 50,000, type of catalyst (2-way or 3-way), carbureted or fuel-injected vehicles, and high or normal emitting vehicles.

After calculating the proportion of vehicles in each of the 20 speed bins and 34 power bins for each segment of each link and constructing 
Figure 1: TRANSIMS Emissions Estimator Framework

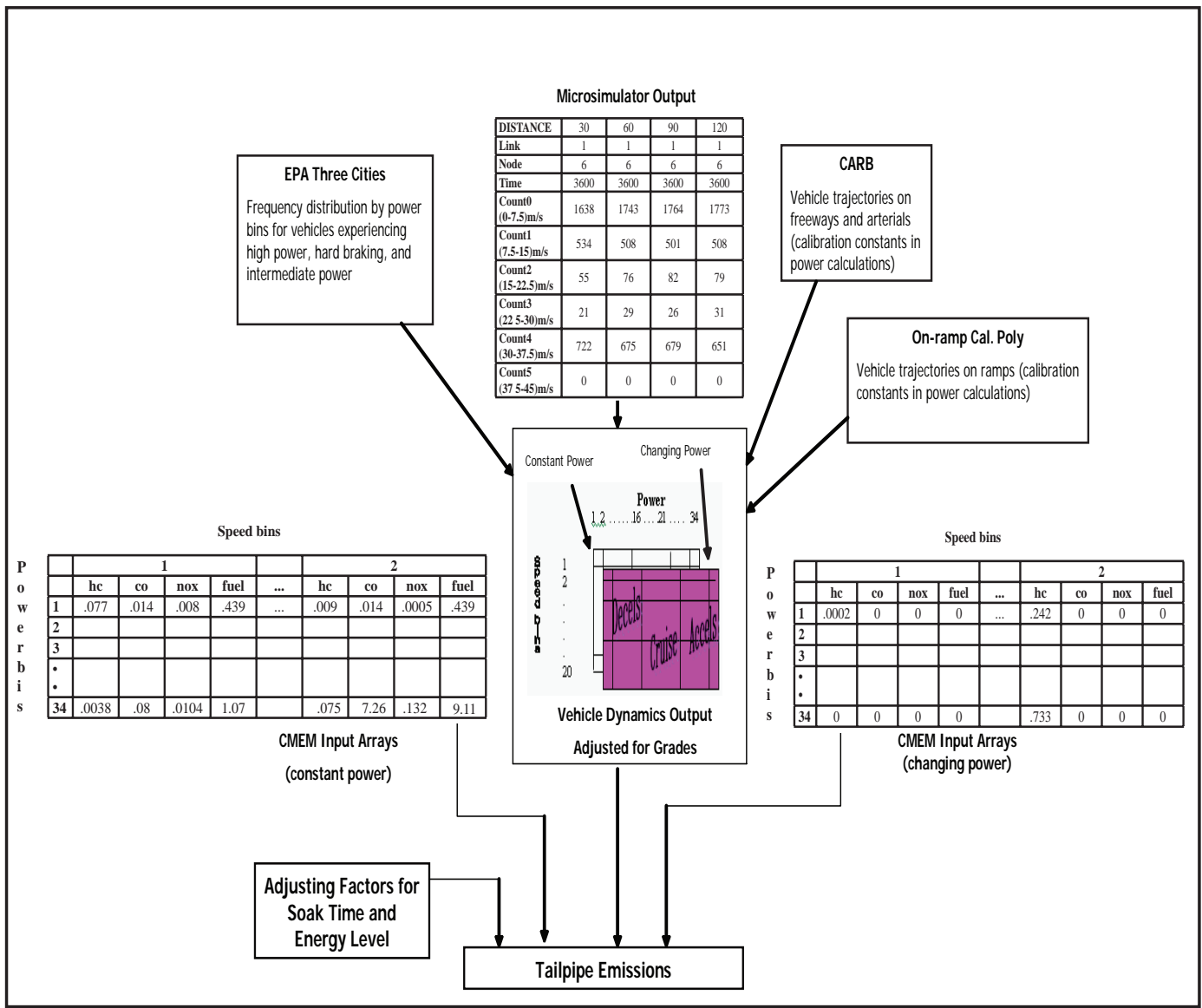

two speed-power matrices - one for constant power and another for the distribution curve for the difference in vehicle power - these matrices are multiplied by the corresponding CMEM emission arrays. The obtained matrices for the constant power and for the difference in emissions between constant power trajectories and those with the same speed and power, but with a step change in power, are summed up. The CMEM arrays give the composite vehicle emissions in terms of $\mathrm{HC}, \mathrm{CO}, \mathrm{NOx}$, and fuel consumption in 4-mph speed bins and 10-mph squared per second power bins. The street grades are incorporated into the analysis by using different CMEM emissions arrays for each street grade classification.

Finally, the calculated values are adjusted for soak-time and energy level by multiplying them by the pollutant adjustment factors. The pollutant adjustment factors are calculated from the converted Microsimulator output and the
CMEM emission rates for one-hour soak-time versus zero soak-time. The emission rates or multipliers that are computed for eight energy groups represent the ratio of emissions for vehicles linked in the group to the emissions of a vehicle with the same driving pattern pertaining to a completely warmed-up engine and catalyst. The converted Microsimulator output is the number of vehicles entering each link over each hour grouped by soak-time and by the integrated velocity-acceleration product. The velocity-acceleration product, which presents the eight energy levels, is used as a surrogate for fuel consumption to give the engine and catalyst a warm-up level. Soak-time is the time the engine was off before the start of the current trip. Four different soak-times are used (e.g., no soak, short soak, medium soak, and long soak-time). A more detailed explanation of the TRANSIMS' Emissions Estimator is presented in Hobeika et al. (2003). 
The inputs from the Microsimulator are the most important inputs to the Emissions Estimator including information about vehicles' speeds and energy levels. In this study, the velocity summary file was obtained from the collected OEM data rather than the Microsimulator's output. The field data were converted to the Microsimulator's output data format and were fed to this module. The data consist of the number of vehicles in each velocity bin in each 30-meter segment on the test link, summed up over 3600 seconds.

\section{Comparison of TRANSIMS, MOBILE6, CMEM, AND VT- MICRO EMISSIONS with OEM Data}

This comparative study used a portable on-road emission measurement device that is designed to collect on-road emission data - OEM-2100 ${ }^{\mathrm{TM}}$, manufactured by Clean Air Technologies International, Inc. This device consists of two analyzers: an engine diagnostic scanner and an on-board computer that provides second-by-second emissions, fuel consumption, engine speed, and engine temperature. The emissions include hydrocarbons (HC), carbon monoxide $(\mathrm{CO})$, carbon dioxide $\left(\mathrm{CO}_{2}\right)$, and oxides of nitrogen $\left(\mathrm{NO}_{x}\right)$. It measures vehicle mass exhaust emissions using vehicle and engine operational data and measuring the concentration of pollutants sampled from the tailpipe during actual on-road driving. The real-time engine and vehicle operational data are obtained by connecting the unit to the OnBoard Diagnostics (OBD) link of the vehicle. Most cars made after 1995 are equipped with an OBD link. A more detailed description of the OEM device can be found in Rakha et al. (2004).

A 1999 Ford Crown Victoria was utilized for collecting field data. The data were gathered along a 16-km path including highway, arterial, and ramp roadways with speed limits varying from $25 \mathrm{mph}$ to $55 \mathrm{mph}$, in Blacksburg, Virginia, as presented in Figure 2. This path, which includes South Main Street, North Main Street, and Route 460, was selected because it is part of the TRANSIMS Blacksburg network. The data collected included 13 repetitions of

\section{Figure 2: The Road Section in the Blacksburg Network Used to Collect OEM Data}

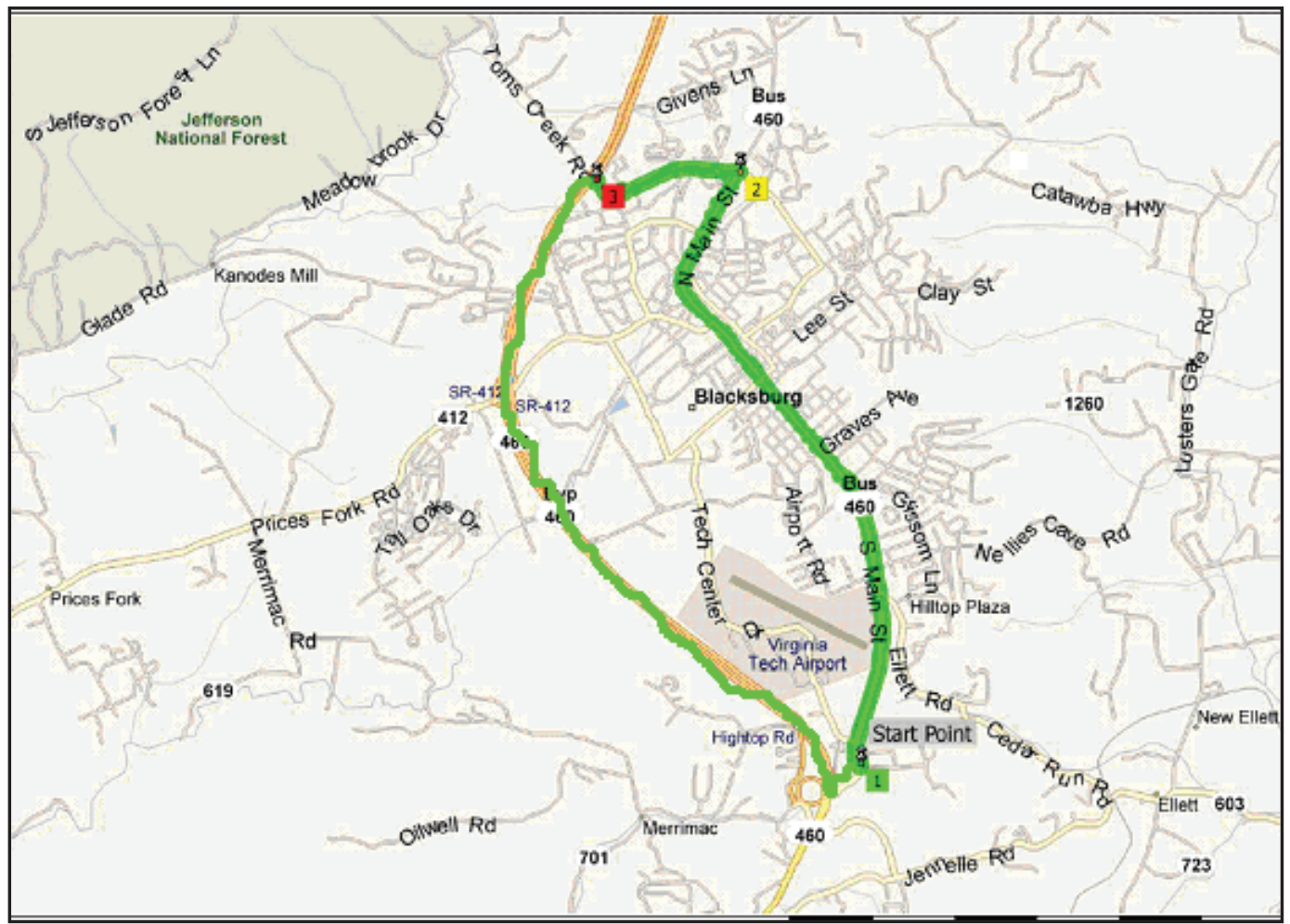


normal driving along the study section. The speed profiles of the 13 runs are presented in Figure 3.

The TRANSIMS Emissions Estimator was run using the above input data. The results of the hydrocarbons, carbon monoxide, oxides of nitrogen, and fuel consumption estimates for each of the 13 runs are recorded in Figure 4. These results show that the estimated hydrocarbons are consistent with the OEM estimation in terms of magnitude.

This model is unlike others that estimate a considerably lower amount for hydrocarbons compared to OEM. It also shows a close variation across the 13 test runs compared to the OEM data. The TRANSIMS estimation of carbon monoxide is almost consistent with the OEM estimation in terms of variation across the 13 runs, while its size is underestimated compared to that calculated using the OEM data. The nitrogen oxide and fuel consumption estimates from TRANSIMS are less than those calculated by the OEM data. They are also inconsistent with the OEM estimation in terms of variation across the 13 runs. Other models' estimations of fuel consumption are consistent with the OEM data in terms of both magnitude and the trend across the runs. Although TRANSIMS uses the CMEM emission arrays as input to its emission estimation procedure,

Figure 3: The Speed Profile of the Test Runs 1 to 13

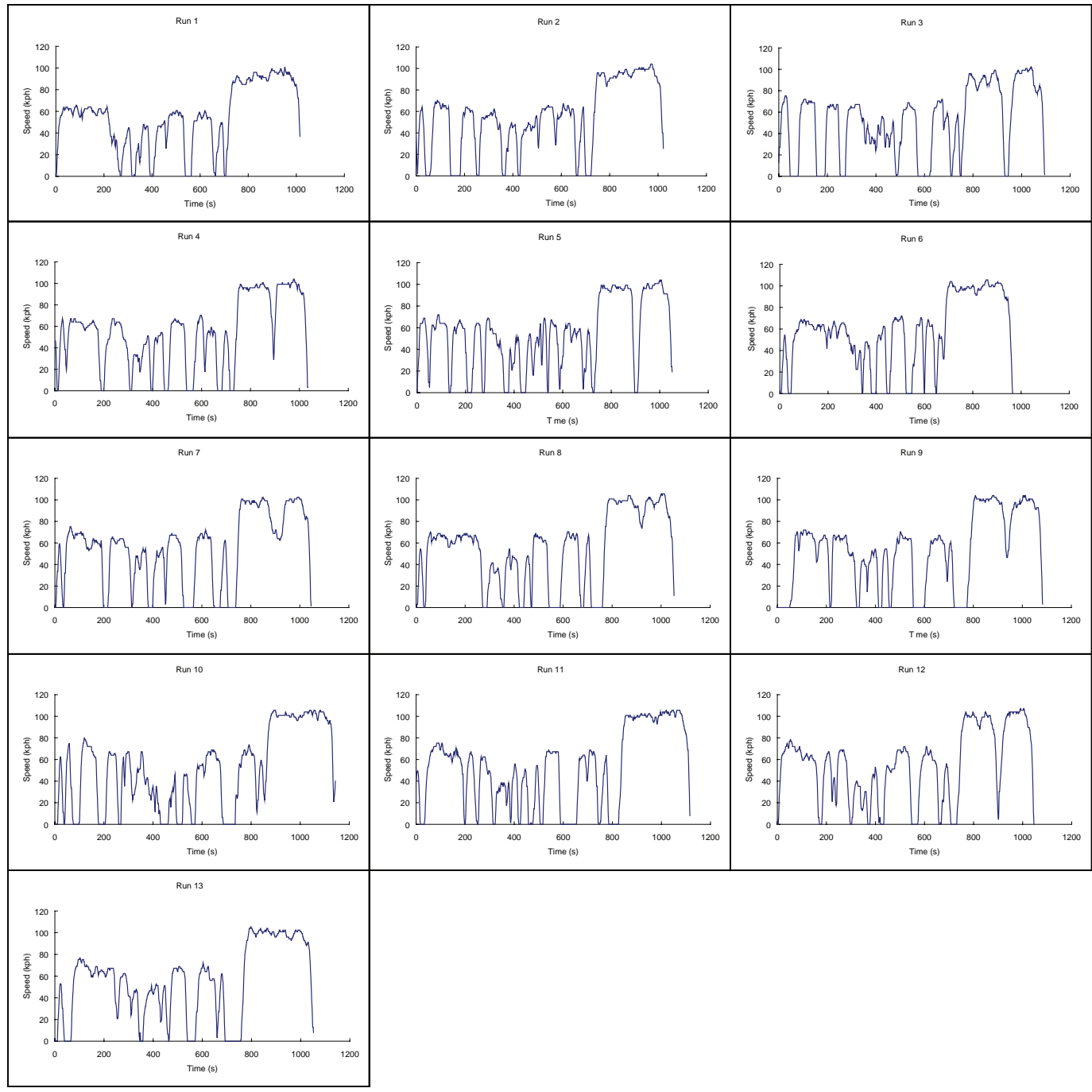


its emission estimates are significantly different from the CMEM estimates - which is attributed to its estimation of vehicle speed trajectories. Consequently, it can be argued that in cases where the TRANSIMS estimates resulted in a better match with the field data that these were caused by the aggregation of the vehicle speed profile and not attributed to better modeling of vehicle emissions.

The results also demonstrate that MOBILE6 is not sensitive to differences in trip speed profiles across the 13 runs given that the average speed remained fairly constant across the runs. The VT-Micro trends are more consistent with the OEM trends across the various runs, while TRANSIMS' estimates are closer to those estimated by OEM compared to the other models.

The differences in the emission estimates between those produced by TRANSIMS and the field data can be attributed to two factors. First, it could be argued that a direct comparison with field measurements for a single vehicle against aggregated composite vehicle emission estimates may not be a fair comparison. However, this study demonstrates consistency in cyclic variations across the two scenarios. Specifically, the VT-Micro model (Rakha et al. 2004a) calibrated against the Crown Victoria vehicle was compared to the VT-Micro LDV5 vehicle emission and fuel consumption estimates. Although the absolute emission and fuel consumption estimates were different, the trends of both models were almost identical. Second, TRANSIMS uses the CMEM emission arrays as one of the main inputs of its emissions estimator, while there is no procedure to calculate these two arrays, the arrays developed for the Portland network using CMEM1.2 are being used as an input to the Emissions Estimator module. It may be more precise if research could provide the distribution of vehicles for the study network as input. Then, the Emissions Estimator would calculate the two arrays for that specific network rather than using the same array for all networks. This study attempted to re-produce the two arrays using CMEM, but was unable to do so due to ambiguity in the procedure for constructing the two arrays required by the developers of TRANSIMS.

Table 1 presents a comparison of the models and their results. The models are either aggregated (A) or microscopic (M). They either use speed or speed and acceleration to calculate emissions. They also utilize regression models or vehicle operating modes to estimate emissions. Finally, the table reports whether the overall trend and magnitude of the emissions estimated in each model follow those estimated by the OEM data.

Table 1: Comparison of Emissions Models

\begin{tabular}{|c|c|c|c|c|c|c|}
\hline Model & $\mathbf{A} / \mathbf{M}$ & Speed & Acceleration & Method & Trend & Magnitude \\
\hline Mobile6 & A & $\sqrt{ }$ & - & Regression & $\mathrm{x}$ & $\mathrm{x}$ \\
\hline CMEM & M & $\sqrt{ }$ & $\sqrt{ }$ & $\begin{array}{c}\text { Vehicle Operating } \\
\text { modes }\end{array}$ & $\mathrm{x}$ & $\mathrm{x}$ \\
\hline VT-Micro & M & $\sqrt{ }$ & $\sqrt{ }$ & Regression & $\sqrt{ }$ & $\mathrm{x}$ \\
\hline TRANSIMS & M & $\sqrt{ }$ & $\sqrt{ }$ & $\begin{array}{c}\text { Vehicle Operating } \\
\text { modes }\end{array}$ & $\mathrm{x}$ & $\sqrt{ }$ \\
\hline
\end{tabular}

$\mathrm{X}=$ refers that the model does not follow the OEM data. 
Vehicle Emissions

Figure 4: The Emission Estimate in TRANSINS, MOBILE6, CMEM, VT-Composite Versus the OEM Data
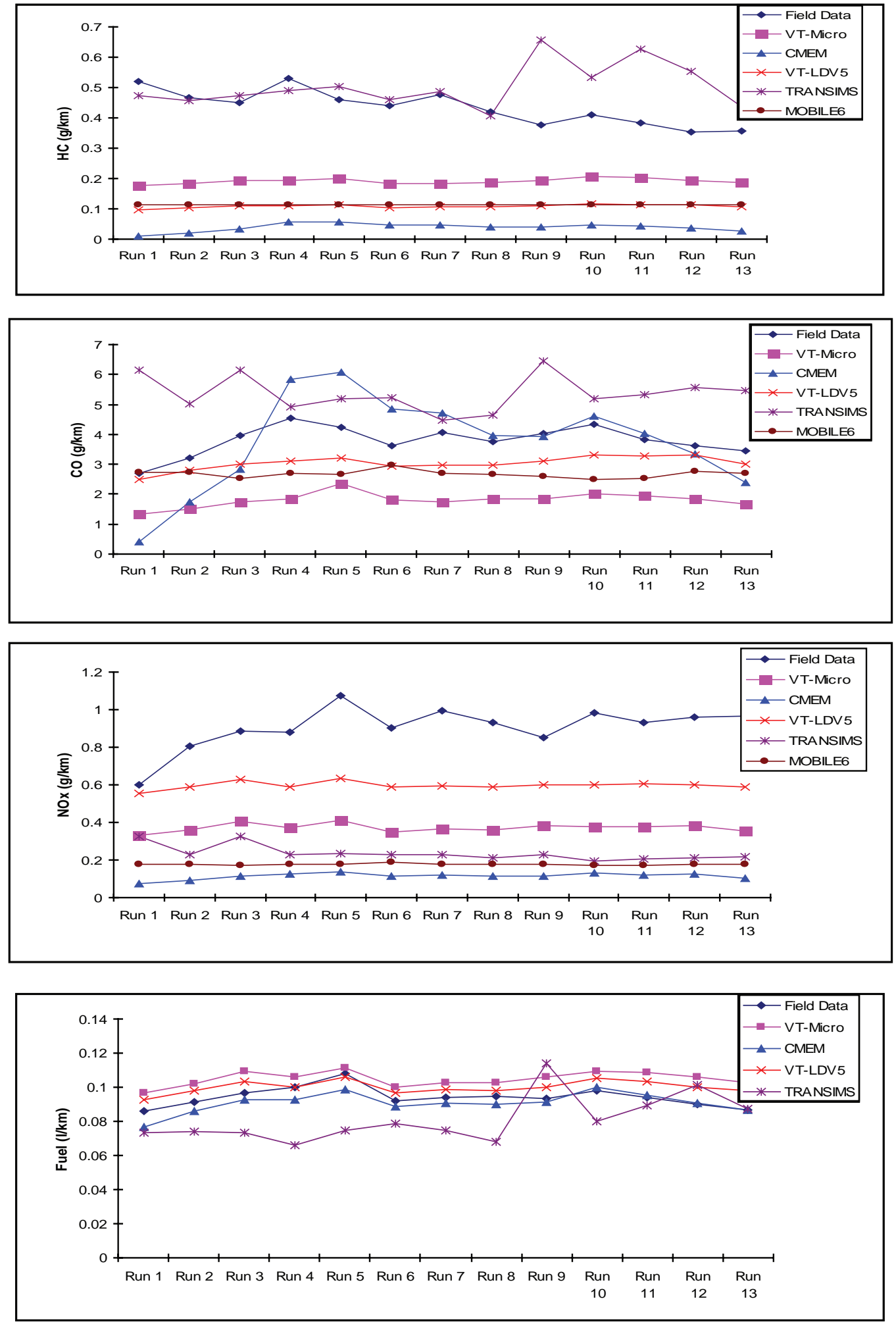


\section{SUMMARY AND CONCLUSIONS}

This paper describes the emission estimation procedures within the TRANSIMS model. TRANSIMS relies heavily on three sets of empirical data pertaining to power demands, which are CMEM, the EPA three-cities data, and CARB. It addresses most existing problems in the traditional emissions models that use an aggregate representation of travel behavior and estimates emissions based on typical driving cycles using vehicle miles traveled and average speeds. TRANSIMS considers coldstarts, enrichment cycles, grades, and vehicle malfunctioning in emission calculations. TRANSIMS also integrates the transportation modeling procedure and the air quality modeling procedure to estimate emissions directly based on vehicle movements on a transportation network.

The emissions estimated by TRANSIMS are compared with the OEM emissions estimates. The results show that TRANSIMS' emissions almost reflect the differences across drive cycles that were observed in field measurements for carbon monoxide and hydrocarbons, but not for oxides of hydrogen and fuel consumption. The results of the comparison between the OEM data and the emissions estimates of TRANSIMS, CMEM, and the VT-Micro models are summarized as follows.

- TRANSIMS' emission magnitudes are more consistent with the in-field emission measurements when compared with the other models. However, this is attributed to the aggregation of the speed profiles and not a result of more accurate emission modeling.

- The VT-Micro model emission trends appear to be more consistent with the infield emission measurements as compared with the other models.

- MOBILE6 is not sensitive to different speed profiles and presents almost a flat trend across different runs.

- Unlike TRANSIMS, other models underestimate the emission magnitudes when compared with the field data.

- Unlike other models, TRANSIMS does not yield a consistent estimation of fuel consumption, both in trend and magnitude, with the OEM data. Thus, its calculation procedure should be reviewed.

- The emissions estimation procedure in TRANSIMS could be modified by adding a sub-module to calculate the CMEM emission arrays for each network by providing the distribution of vehicles for the specific network.

- The TRANSIMS emission model appears to be appropriate for use with the environmental evaluations for small projects; however, it requires modification as earlier mentioned.

\section{References}

Ahn, K., H. Rakha, A. Trani, and M. Van Aerde. "Estimating Vehicle Fuel Consumption and Emissions Based on Instantaneous Speed and Acceleration Levels.” Journal of Transportation Engineering 128(2), (2002): 182-190.

Barth, M., T. Younglove, T. Wenzel, G. Scora, F. An, M. Ross, and J. Norbeck. “Analysis of Modal Emissions for a Diverse in-use Vehicle Fleet.” Transportation Research Record: Journal of the Transportation Research Board, No. 1587, TRB, National Academy of Science, Washington, D.C., (1997):73-84.

Barth, M., F. An, T. Younglove, G. Scora, C. Levine, M. Ross, and T. Wenzel. Comprehensive Modal Emission Model (CMEM), version 2.0 user's guide, Riverside, CA, 2000.

Hobeika, A. G. H. D. Sherali, C. Jeenanantua, and M. Jeihani, TRANSIMS Short Course

Documents, Virginia Polytechnic Institute and State University, Blacksburg, VA, 2003. 
Oliver, W. R., R. J. Dickson, and L. Bruckman. "Development of the SCAQS High-Resolution Emissions Inventory: Assessment of Inventory Uncertainties” in Proceedings of an International Specialty Conference, Southern California Air Quality Study, Data Analysis, VIP-26, Air \& Waste Management Association, Pittsburgh, Pennsylvania, (1993): 62-73.

Portland Study Report, Postprocessing for Environmental Analysis, Volume 5, Los Alamos National Laboratory, LA-UR-01-5715, Los Alamos, NM, 2001.

Rakha H. and Ahn K. "INTEGRATION Modeling Framework for Estimating Mobile Source Emissions.” Journal of Transportation Engineering 130(2), (2004): 183-193.

Rakha, H., K. Ahn, I. El-Shawarby, and S. Jang. "Emission Model Development Using Invehicle On-Road Emission Measurement.” Environmental Quality and Analysis. CD-ROM. Transportation Research Board, National Research Council, Washington, D.C., (2004a): 1-12.

Rakha, H., K. Ahn, and A. Trani. "Development of VT-Micro Model for Estimating Hot Stabilized Light Duty Vehicle and Truck Emission.” Transportation Research Part D-Transport and Environment 9(1), (2004b): 49-74.

Rakha, H., M. Van Aerde, K. Ahn, and A.A. Trani. "Requirements for Evaluating Traffic Signal Control Impacts on Energy and Emissions Based on Instantaneous Speed and Acceleration Measurements.” Transportation Research Record 1738, (2000): 56-67.

Roden, D. B. “The Impact of Regional Simulation on Emission Estimates.” Environmental Quality and Analysis. CD-ROM. Transportation Research Board, National Research Council, Washington, D.C., (2005): 1-19.

TRANSIMS homepage. Los Alamos National Laboratory, Los Alamos, NM. http://transims.tsasa. lanl.gov.

TRANSIMS Documents, Los Alamos National Laboratory, LA-UR-00-1725, TRANSIMS 3.0, Los Alamos, NM, 2000, Volume 3, Chapter 7.

Williams, M. D., G. Thayer, L. L. Smith, and M. J. Barth, Recent Developments in the TRANSIMS Approach to Emissions Estimation. TRANSIMS Documents, Los Alamos National Laboratory, LA-UR-00-388, Los Alamos, NM, 2000.

Williams, M. D., G. Thayer, and L. L. Smith, The TRANSIMS Approach to Emissions Estimation. TRANSIMS Documents, Los Alamos National Laboratory, LA-UR-99-471, Los Alamos, NM, 2000 .

Mansoureh Jeihani received her B.S. degree in computer engineering from Iran National University (Shahid Beheshti), Tehran, Iran, in 1995, an M.S. degree in socio-economics systems engineering from the Institute for Research in Planning and Development (IRPD), Tehran, Iran, in 1998, an M.A. degree in economics from Virginia Tech in 2001, and a Ph.D. degree in transportation systems engineering from Virginia Tech in 2004. She is currently working as a senior associate at Resource Systems Group, Inc. She is a member of ASCE and ITE.

Kyoungho Ahn is a senior research associate in the Center for Sustainable Mobility at the Virginia Tech Transportation Institute. His main areas of research include transportation environmental modeling, traffic flow theory, and traffic modeling and simulation. He is also knowledgeable in the area of operations research, urban planning, and transportation planning. He has authored/coauthored a number of fully-refereed journal publications. He has developed the VT-Micro emission models. He is a member of ASCE and TRB. 
Antoine G. Hobeika is a professor in the Department of Civil and Environmental Engineering at Virginia Polytechnic Institute and State University. His research interests lie in transportation planning and operations, intelligent transportation systems, transportation emergency management, and transportation economics. In 1988, he established the University Center for Transportation Research at Virginia Tech and he directed the FHWA ITS Center of Excellence which he was able to attract in 1993. He is a member of ITE, ASCE, TRB, and ITS-America. He published several papers in these organizations' journals and in other scientific journals.

Hanif D. Sherali is the W. Thomas Rice Endowed Chaired Professor of Engineering in the Industrial and Systems Engineering Department at Virginia Polytechnic Institute and State University. His area of research interest is in discrete and continuous optimization, with applications to location, transportation, and engineering design problems. He has published several papers in operations research journals, has co-authored five books in this area, and serves on the editorial board of eight journals. He is a member of the National Academy of Engineering.

Hesham A. Rakha is an associate professor at the Charles Via Jr. Department of Civil and Environmental Engineering and the director of the Center for Sustainable Mobility at the Virginia Tech Transportation Institute. He is a professional engineer in Ontario and is a member of the Institute of Transportation Engineers (ITE), the American Society of Civil Engineers (ASCE), the Institute of Electrical and Electronics Engineers (IEEE), and the Transportation Research Board (TRB). His main areas of research include traffic flow theory, traffic modeling and simulation, dynamic traffic assignment, traffic control, energy and environmental modeling, and safety modeling. He has published extensively in these research areas. 